\title{
Capacidad fitorremediadora de cinco especies altoandinas de suelos contaminados con metales pesados
}

\author{
Phytoremediation capacity of five high andean species from soils contaminated with heavy \\ metals
}

\author{
Enoc Jara-Peña ${ }^{1,2}$, José Gómez ${ }^{1,2}$, Haydeé Montoya ${ }^{2}$, Magda Chanco ${ }^{2}$ Mauro Mariano ${ }^{3}$ y Noema Cano ${ }^{2}$
}

1 Laboratorio de Fitología Aplicada, Facultad de Ciencias Biológicas, Universidad Nacional Mayor de San Marcos, Ciudad Universitaria, Av. Venezuela 3400, Lima 1, Perú. / Apdo. 11-0058. Lima 11, Perú.

2 Museo de Historia Natural. Laboratorio de Simbiosis Vegetal, Universidad Nacional Mayor de San Marcos. Av. Arenales 1256, Lima 11, Perú.

3 Laboratorio de Investigación de Fauna Dulceacuícola, Facultad de Ciencias Biológicas, Universidad Nacional Mayor de San Marcos.

Email Enoc Jara-Peña: ejarap@unmsm.edu.pe Email José Gómez: jgomezc@unmsm.edu.pe Email Haydeé Montoya: haydmon@yahoo.com Email Magda Chanco: mchancoestela@yahoo.es Email Mauro Mariano: mmarianoa@unmsm.edu.pe Email Noema Cano: noemacano@hotmail.com

\section{Citación:}

Jara-Peña E., J. Gómez, H. Montoya, M. Chanco, M. Mariano \& N. Cano. 2014. Capacidad fitorremediadora de cinco especies altoandinas de suelos contaminados con metales pesados. Revista peruana de biología 21(2): 145 - 154 (Octubre 2014). doi: http://dx.doi.org/10.15381/rpb.v21i2.9817

Fuentes de financiamiento:

El presente trabajo fue financiado por:

Vicerrectorado de Investigaciones de la Universidad Nacional Mayor de San Marcos, Proyecto No. 121001141

\section{Resumen}

La fitorremediación consiste en el uso de plantas para remediar in situ suelos, sedimentos, agua y aire contaminados por desechos orgánicos, nutrientes o metales pesados, eliminando los contaminantes del ambiente o haciéndolos inocuos. El trabajo fue realizado en condiciones de invernadero en el distrito de Lachaqui, provincia de Canta, región Lima, de octubre de 2011 a octubre de 2012. Fueron evaluados veinte tratamientos con un diseño factorial completo 5 x 4: 5 especies alto andinas, y 4 sustratos con $30 \%, 60 \%, 100 \%$ de relave de mina (RM) y suelo sin RM. La producción de biomasa disminuyó significativamente en Solanum nitidum, Brassica rapa, Fuertesimalva echinata y Urtica urens y Lupinus ballianus, con el tratamiento de $100 \%$ de relave de mina. La mayor eficiencia de acumulación de plomo y zinc fue obtenida en las raíces de Fuertesimalva echinata con el tratamiento de $100 \%$ de relave de mina, obteniendo $2015.1 \mathrm{mg}$ de plomo $\mathrm{kg}^{-1} \mathrm{MS}$ y $1024.2 \mathrm{mg}$ de zinc kg-1 MS. En las raíces de $L$. ballianus fue obtenida la más alta acumulación de cadmio, con una concentración de 287.3 $\mathrm{mg} \mathrm{kg}^{-1} \mathrm{MS}$ con el tratamiento de $100 \%$ de relave de mina. Fuertesimalva echinata presentó el mayor índice de tolerancia (IT) al tratamiento de $100 \%$ de relave de mina, con un IT de $41.5 \%$, pero, S. nitidum y L. ballianus presentaron el mayor IT al tratamiento de $60 \%$ de relave de mina con IT de $68.5 \%$ y 67.9 .

Palabras claves: fitorrestauración; plantas peruanas; ecosistemas andinos; fitoestabilización; relave de mina.

\section{Abstract}

Phytoremediation consist in the use of plants to recover soil, sediments, water and air in situ, which have been contaminated by organic waste, nutrients or heavy metals, by removing pollutants from the environment or making them harmless. The evaluation work was conducted under greenhouse conditions in Lachaqui, Canta, Lima, from October 2011 to October 2012. Twenty treatments were evaluated with a $5 \times 4$ factorial design: 5 high elevation Andean species, and 4 substrates with $30 \%, 60 \%, 100 \%$ mine waste (MW) and tailings soil without MW. In Solanum nitidum, Brassica rapa, Fuertesimalva echinata, Urtica urens and Lupinus ballianus, the biomass production decrease significantly with the of $100 \%$ mine waste treatment (MW). The major efficiency accumulation of lead and zinc was obtained in the roots of Fuertesimalva echinata with $100 \%$ MW treatment, obtaining $2015.1 \mathrm{mg}$ of lead kg-1 DM and $1024.2 \mathrm{mg}$ of zinc kg-1 DM, this specie presented the major tolerance index ( $\mathrm{TI}$ ) to $100 \% \mathrm{MW}$ treatment, with $41.5 \% \mathrm{TI}$. The highest cadmium accumulation was obtained in the roots of $L$. ballianus, with a concentration of $287.3 \mathrm{mg} \mathrm{kg}-1$ DM with $100 \% \mathrm{MW}$ treatment, this species also had a $67.9 \%$ $\mathrm{TI}$ in $60 \% \mathrm{MW}$ treatment. And finally, S. nitidum presented a $68.5 \% \mathrm{TI}$ in $60 \% \mathrm{MW}$ treatment. Keywords: phytorestoration; peruvian plants; andean ecosystems; phytostabilization; mine waste.

\section{Información sobre los autores:}

Los autores declaran haber partcipado en el presente trabajo realizando: EJ: diseño, ejecución y redacción; JG: diseño, ejecución y redacción; HM: diseño, ejecución;NC: diseño y redacción, MA: diseño, ejecución, MCh: diseño, indentificación de muestras.

$\begin{array}{ll}\text { Presentado: } & \text { 22/05/2013 } \\ \text { Aceptado: } & \text { 09/06/2013 } \\ \text { Publicado online: } & \text { 07/10/2014 }\end{array}$

Journal home page: http://revistasinvestigacion.unmsm.edu.pe/index.php/rpb/index

(c) Los autores. Este artículo es publicado por la Revista Peruana de Biología de la Facultad de Ciencias Biológicas, Universidad Nacional Mayor de San Marcos. Este es un artículo de acceso abierto, distribuido bajo los términos de la Licencia Creative Commons Atribución-NoComercial-Compartirlgual 4.0 Internacional.(http://creativecommons.org/licenses/by-nc-sa/4.0/), que permite el uso no comercial, distribución y reproducción en cualquier medio, siempre que la obra original sea debidamente citadas. Para uso comercial, por favor póngase en contacto con editor.revperubiol@gmail.com. 


\section{Introducción}

En los ecosistemas andinos situados por encima de los $3300 \mathrm{~m}$ de altitud se forman las cabeceras de las cuencas de las Vertientes Occidental y Oriental de los Andes, aquí podemos encontrar praderas de pastizales, parches de bosques, matorrales y bofedales (Young et al. 1997), muchos de ellos amenazados por la minería y actividades asociadas. Las actividades mineras depositan sus residuos con metales pesados en la superficie del entorno minero causando la contaminación del suelo, y representando un problema ambiental de gran preocupación mundial (Alkorta et al. 2010).

En general, los suelos originales de las minas se degradan o se pierden irreversiblemente, generando nuevos suelos modificados formados por materiales poco aptos para el desarrollo de procesos biológicos (Becerril et al. 2007). Las consecuencias directas de esta contaminación del suelo son la desaparición de la vegetación, pérdida de su productividad y disminución de la biodiversidad; indirectamente se menciona la contaminación del aire, y aguas superficiales y subterráneas (Wong 2003).

Sin embargo, existen las denominadas plantas metalófitas, que han desarrollado los mecanismos fisiológicos para resistir, tolerar y sobrevivir en suelos degradados por actividades mineras (Becerril et al. 2007). Estas especies pueden restringir la absorción los metales o translocarlos hacia las hojas o absorberlo y acumularlo activamente en su biomasa aérea (Baker \& Proctor 1990). Algunas plantas modifican las condiciones de la rizósfera produciendo exudados radiculares o la alterando el $\mathrm{pH}$ (Adriano 2001, Wenzel et al. 2003). Los grados de acumulación metálica van desde trazas hasta más del $1 \%$ de la materia seca de la planta (Diez 2008).

Actualmente para la recuperación de suelos contaminados con metales pesados se tienen varias tecnologías (Diez 2008), estas generalmente recurren al uso de plantas metalófitas que pueden utilizarse en los procesos de fitorrestauración y fitorremediación para recuperar sedimentos y aguas contaminadas por metales pesados, eliminando los contaminantes del ambiente o haciéndolos inocuos (Salt et al. 1998).

Por otro lado, están siendo investigadas las denominadas plantas hiperacumuladoras, aquellas capaces de acumular más de $1000 \mathrm{mg}$ de Níquel por kilogramo de materia seca, o más de $10000 \mathrm{mg} \mathrm{kg}^{-1}$ de $\mathrm{Mn}$ y Zn, más de $1000 \mathrm{mg} \mathrm{kg}^{-1}$ de Co, $\mathrm{Cu}, \mathrm{Ni}$ y $\mathrm{Pb}$ y más de $100 \mathrm{mg} \mathrm{kg}^{-1}$ de Cd (Brooks et al. 1977, Baker et al. 2000), que podrían usarse en las diferentes técnicas de fitorremediación.

El presente trabajo informa los resultados de un experimento que evalúa la capacidad de fitorremediación de cinco plantas andinas: Solanum nitidum, Brassica rapa, Fuertesimalva echinata, Urtica urens y Lupinus ballianus, en suelos contaminados con plomo, zinc y cadmio.

\section{Material y métodos}

El experimento fue realizado en condiciones de invernadero

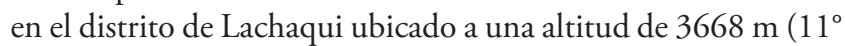
33'11'S, 76³7'32”W), provincia de Canta, región Lima, en el periodo de octubre 2011 a octubre 2012. La temperatura media mensual y la humedad relativa media mensual fueron registradas utilizando un Higrotermógrafo digital, marca VWR modelo 62344-734, cuyos valores se presentan en la Tabla 1.
La unidad experimental fue una maceta de $19 \mathrm{~cm}$ de diámetro y $5 \mathrm{~kg}$ de capacidad. Como sustrato fueron utilizados suelo libre de metales pesados, cuyas características físico-químicas se señalan en la Tabla 2 y relave de mina polimetálico $(\mathrm{RM})$, que fue obtenido de una concentradora polimetálica, en la localidad de Yani, distrito de Huamantanga, Provincia de Canta y sus características químicas se indican en la Tabla 3. Posteriormente, se procedió con la adición de los sustratos en las macetas, según los tratamientos correspondientes.

Las semillas de Solanum nitidum Ruiz \& Pav. (Solanaceae) fueron colectados en el distrito de Aquia (Departamento de Ancash). Las semillas Brassica rapa L.(Brassicaceae), Fuertesimalva echinata (C.Presl) Fryxell (Malvaceae), Urtica urens L.(Urticaceae) y Lupinus ballianus C.P. Sm (Fabaceae), fueron colectadas en el distrito de Lachaqui.

Las semillas de estas cinco especies fueron sembradas a razón de 6 semillas viables de cada especie en cada maceta, y según los tratamientos correspondientes. Los sustratos fueron regados con agua potable y se mantuvieron en su capacidad de campo durante todo el experimento. Las semillas de las especies iniciaron su germinación entre los 21 y los 30 días después de la siembra.

Se utilizó un diseño experimental completamente al azar, fueron evaluados 20 tratamientos con 5 repeticiones por tratamiento. Los tratamientos se generaron de un factorial completo $5 \times 4$, en donde 5 indica las cinco especies altoandinas, y 4 indica los cuatros sustratos (30\% de relave de mina, 60\% y $100 \%$ de relave de mina, y suelo sin relave (control). El tratamiento 30\% de relave de mina consistió de una mezcla de $1.5 \mathrm{~kg}$ de RM y $3.5 \mathrm{~kg}$ de suelo (853.0 $\mathrm{mg}$ de plomo por kilogramo de suelo, $1134.0 \mathrm{mg}$ de zinc por kilogramo de suelo y $24.0 \mathrm{mg}$ de cadmio por kilogramo de suelo). El tratamiento de $60 \%$ de relave de mina consistió de una mezcla de $3.5 \mathrm{~kg}$ de RM y $1.5 \mathrm{~kg}$ de suelo (1707.6 mg de plomo por kilogramo de suelo y 2268.0 $\mathrm{mg}$ de zinc por kilogramo de suelo y $48.1 \mathrm{mg}$ de cadmio por kilogramo de suelo).

Tabla 1. Tempertura media mensual y humedad relativa registradas en el invernadero, desde octubre de 2011 hasta octubre de 2012, en el distrito de Lachaqui, provincia de Canta, región Lima.

\begin{tabular}{ccc}
\hline Meses & $\begin{array}{c}\text { Tempertura media } \\
\text { mensual }\left({ }^{\circ} \mathrm{C}\right)\end{array}$ & $\begin{array}{c}\text { Humedad Relativa } \\
\text { media mensual (\%) }\end{array}$ \\
\hline Oct-2011 & 22.8 & 57.6 \\
Nov-2011 & 24.8 & 56 \\
Dic-2011 & 21.9 & 61.4 \\
Ene-2012 & 16.4 & 72 \\
Feb-2012 & 19.6 & 80.5 \\
Mar-2012 & 18.9 & 81.5 \\
Abr-2012 & 22.5 & 59.1 \\
May-2012 & 28.7 & 47.5 \\
Jun-2012 & 17.6 & 38 \\
Jul-2012 & 13.7 & 31 \\
Ago-2012 & 14.1 & 33 \\
Sep-2012 & 24.7 & 39 \\
Oct-2012 & 21.6 & 43 \\
\hline
\end{tabular}


Tabla 2. Características físico-químicas del suelo utilizado en el experimento realizadas en el distrito de Lachaqui, provincia de Canta, región Lima.

\begin{tabular}{|c|c|c|c|c|}
\hline \multicolumn{2}{|l|}{ Determinación } & \multirow{2}{*}{$\begin{array}{c}\text { Valor } \\
60\end{array}$} & \multirow[t]{2}{*}{ Metodología } & \multirow[t]{2}{*}{ Interpretación } \\
\hline Arcilla & $(\%)$ & & & \\
\hline Limo & $(\%)$ & 28 & Hidrómetro de & Franco arenoso \\
\hline Arena & $(\%)$ & 60 & Bouyoucos & \\
\hline Capacidad de campo & $(\%)$ & 23.2 & Olla de presión & - \\
\hline pH (Relación agua 1:1) & & 6.72 & Potenciometría & Ligeramente ácido \\
\hline Conductividad Eléctrica & $\left(\mathrm{dS} \mathrm{m}^{-1}\right)$ & 1.1 & Potenciometría & No salino \\
\hline Materia orgánica & $(\%)$ & 5.6 & Walkley y Black & Alto \\
\hline Capacidad de Intercambi & & 26.4 & & \\
\hline$P$ disponible & $\left(\mathrm{mg} \mathrm{kg}^{-1}\right)$ & 71.8 & Olsen & Medio \\
\hline K disponible & $\left(\mathrm{mg} \mathrm{kg}^{-1}\right)$ & 1948 & & Alto \\
\hline $\mathrm{K}$ intercambiable & $\left(\right.$ cmoles $\left.^{+} \mathrm{kg}^{-1}\right)$ & 3.4 & & Medio \\
\hline Ca intercambiable & $\left(\right.$ cmoles $\left.^{+} \mathrm{kg}^{-1}\right)$ & 16.7 & $\mathrm{CH}_{3} \mathrm{COONH}_{4} 1 \mathrm{~N}$ & Medio \\
\hline Mg intercambiable & $\left(\right.$ cmoles $\left.^{+} \mathrm{kg}^{-1}\right)$ & 3.0 & & Medio \\
\hline Na intercambiable & $\left(\mathrm{cmoles}^{+} \mathrm{kg}^{-1}\right)$ & 3.2 & & -- \\
\hline $\mathrm{Zn}$ & $\left(\mathrm{mg} \mathrm{kg}^{-1}\right)$ & 287.5 & DTPA & Alto \\
\hline $\mathrm{Cu}$ & $\left(\mathrm{mg} \mathrm{kg}^{-1}\right)$ & 39.5 & DTPA & Medio \\
\hline
\end{tabular}

Fuente: Laboratorio de Suelos, Facultad de Agronomía. Universidad Nacional Agraria La Molina.

Para la evaluación de la biomasa ( $\mathrm{g}$ ) de las cinco especies, fueron utilizadas plantas completas cosechadas a los 12 meses luego de instalado el experimento. Luego las muestras fueron secadas en una estufa a $70{ }^{\circ} \mathrm{C}$ y hasta peso constante, de allí enfriadas en un desecador y pesadas en una balanza de precisión.

De igual modo, para determinar la acumulación de plomo, zinc y cadmio, las muestras de plantas fueron cosechadas a los 12 meses de iniciado el experimento y separadas por órganos. En el laboratorio, estos metales fueron determinados en hojas y tallos (Rascio \& Navari-Izzo 2011), considerando que las especies vegetales hiperacumuladoras acumulan metales en estos órganos, y en raíces (Barceló \& Poschenrieder 2003) considerando que las plantas fitoestabilizadoras acumulan los metales en este órgano. En seguida las muestras de cada especie vegetal fueron separadas en hojas y tallos, y en raíces. Posteriormente las muestras fueron secadas en una estufa a $60{ }^{\circ} \mathrm{C}$ hasta peso constante, molidas y luego digeridas con $\mathrm{HNO}_{3}+\mathrm{HCl}$ en un digestor de calor para obtener un extracto (Allan 1971). Las lecturas de las concentraciones de estos elementos fueron determinadas en un Espectrofotómetro de Absorción Atómica, en el Laboratorio de
Tabla 3. Concentración de metales pesados del relave polimetálico utilizado en el experimento, en el distrito de Lachaqui, provincia de Canta, región Lima.

\begin{tabular}{llcl}
\hline Determinación & & Valor & Metodología \\
\hline Zinc total & $\left(\mathrm{mg} \mathrm{kg}^{-1}\right)$ & 3780.0 & Digestión húmeda \\
Cobre total & $\left(\mathrm{mg} \mathrm{kg}^{-1}\right)$ & 1440.0 & Digestión húmeda \\
Plomo total & $\left(\mathrm{mg} \mathrm{kg}^{-1}\right)$ & 2846.0 & Digestión húmeda \\
Cadmio total & $\left(\mathrm{mg} \mathrm{kg}^{-1}\right)$ & 48.1 & Digestión húmeda \\
\hline
\end{tabular}

Fuente: Laboratorio de Suelos, Facultad de Agronomía. Universidad Nacional Agraria La Molina.

Suelos de la Facultad de Agronomía de la Universidad Nacional Agraria La Molina.

El índice de tolerancia (IT) a plomo, zinc y cadmio de las especies vegetales fue calculada mediante la relación, en porcentaje, entre la biomasa de la parte aérea (peso seco de hojas y tallos) en un medio contaminado y la biomasa aérea en un medio no contaminado (Watson et al. 2003). Finalmente, ejemplares de las cinco especies evaluadas fueron colectadas por duplicado, las

Tabla 4. Biomasa (gramos) acumulada por las cinco especies altoandinas evaluadas con tratamientos de relave de mina (\%) en el distrito de Lachaqui, provincia de Canta, región Lima.

\begin{tabular}{|c|c|c|c|c|}
\hline \multirow{2}{*}{ Especies } & \multicolumn{4}{|c|}{ Tratamientos (relave de mina) } \\
\hline & $100 \%$ & $60 \%$ & $30 \%$ & Control \\
\hline Solanum nitidum Ruiz \&Pav. & $\S 9.1 \mathrm{~d}$ & $21.5 c$ & $27.4 \mathrm{ab}$ & $31.3 \mathrm{a}$ \\
\hline Brassica rapa $\mathrm{L}$. & $2.5 \mathrm{c}$ & $3.2 \mathrm{bc}$ & $4.3 \mathrm{~b}$ & $6.7 \mathrm{a}$ \\
\hline Fuertesimalva echinata (C.Presl) Fryxel & $2.1 \mathrm{~b}$ & $3.0 \mathrm{bc}$ & $4.1 \mathrm{ab}$ & $5.1 \mathrm{a}$ \\
\hline Urtica urens L. & $1.2 \mathrm{~b}$ & $1.8 \mathrm{~b}$ & $2.1 \mathrm{bc}$ & $3.6 \mathrm{a}$ \\
\hline Lupinus ballianus C.P. Sm. & $2.8 \mathrm{~d}$ & $4.7 \mathrm{c}$ & $6.3 \mathrm{~b}$ & $8.7 \mathrm{a}$ \\
\hline
\end{tabular}

$\S$ Valores con la misma letra en las columnas son estadísticamente iguales (Tukey, a=0.05). 
Tabla 5. Análisis de varianza de la acumulación de plomo, cadmio y zinc en hojas y tallos, y en raíces de cinco especies altoandinas evaluadas con tratamientos de relave de mina (\%) en el Distrito de Lachaqui, Provincia de Canta, Región Lima.

\begin{tabular}{|c|c|c|c|c|c|c|c|c|c|c|c|c|}
\hline \multirow[b]{3}{*}{ Acumulación de metales } & \multicolumn{12}{|c|}{ Tratamientos } \\
\hline & \multicolumn{3}{|c|}{ 100\% Relave de mina } & \multicolumn{3}{|c|}{$60 \%$ Relave de mina } & \multicolumn{3}{|c|}{$30 \%$ Relave de mina } & \multicolumn{3}{|c|}{ Control } \\
\hline & 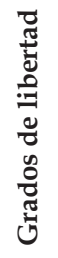 & 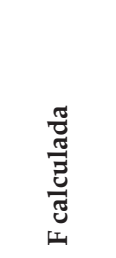 & 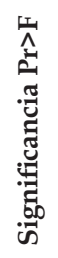 & 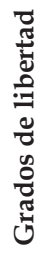 & 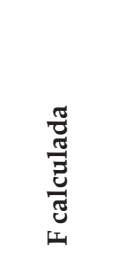 & 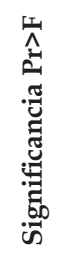 & 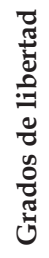 & 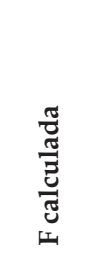 & 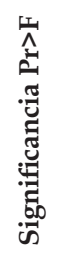 & 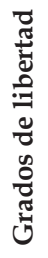 & $\frac{\widetilde{J}}{\underset{\tilde{J}}{\tilde{J}}}$ & \\
\hline Plomo hojas y tallos & 4 & 7108,9 & $* * *$ & 4 & 2281,6 & $* * *$ & 4 & 405 & $* * *$ & 4 & 0,02 & NS \\
\hline Plomo raíces & 4 & $2,5+05$ & $* * *$ & 4 & 93063,6 & $* * *$ & 4 & 2094,5 & $* * *$ & 4 & 4,5 & ** \\
\hline Plomo total & 4 & $1,9 e+05$ & $* * *$ & 4 & 72252,5 & $* * *$ & 4 & 2091,8 & $* * *$ & 4 & 4,4 & * \\
\hline Zinc hojas y tallos & 4 & 1202,4 & $* * *$ & 4 & 1275,9 & $* * *$ & 4 & 160,3 & $* * *$ & 4 & 34,4 & $* * *$ \\
\hline Zinc raíces & 4 & 11050,3 & $* * *$ & 4 & 31423,4 & $* * *$ & 4 & 3867,1 & $* * *$ & 4 & 11,2 & $* * *$ \\
\hline Zinc total & 4 & 8177,6 & $* * *$ & 4 & 24527,5 & $* * *$ & 4 & 2154,7 & $* * *$ & 4 & 15,9 & $* * *$ \\
\hline Cadmio hojas y tallos & 4 & 32,4 & $* * *$ & 4 & 87,6 & $* * *$ & 4 & 33,4 & $* * *$ & 4 & 1,1 & NS \\
\hline Cadmio raíces & 4 & 258,0 & $* * *$ & 4 & 1180,4 & $* * *$ & 4 & 175.7 & $* * *$ & 4 & 8,4 & $* * *$ \\
\hline Cadmio total & 4 & 198,3 & $* * *$ & 4 & 705,1 & $* * *$ & 4 & 167.1 & $* * *$ & 4 & 7,2 & $* * *$ \\
\hline
\end{tabular}

*: Significancia al $0.05 \quad$ **: Significancia al $0.0105 \quad$ ***: Significancia al $0.001 \quad$ NS: No significativa

cuales han sido depositados en el Herbario San Marcos (USM), Museo de Historia Natural.

\section{Resultados}

Biomasa.- Al realizar la prueba de comparación múltiple de medias, por la Prueba de Tukey, se demostró que existen diferencias significativas $(\mathrm{P}<0.050)$ entre tratamientos y al menos uno de ellos es diferente de los demás (Tabla 3). De las cinco especies evaluadas, el mayor valor de biomasa fue obtenido con el tratamiento control. Y comparando los valores de biomasa entre las especies evaluadas, Solanum nitidum acumuló la mayor producción de biomasa $(31.3 \mathrm{~g})$.

Cabe indicar, que Solanum nitidum es una especie arbustiva y las otras especies son plantas herbáceas. Por otra parte, los menores valores de biomasa en las 5 especies evaluadas, fueron obtenidos con el tratamiento de $100 \%$ de relave de mina (Tabla 4).

Acumulación y distribución de plomo, zinc y cadmio.- El análisis de varianza mostró que existen diferencias significativas $(\mathrm{P}<0.05)$ entre los tratamientos e indicó que al menos uno de ellos fue diferente a los demás frente a diversas concentraciones de acumulación de plomo, zinc y cadmio distribuidas tanto en las hojas y tallos, como en las raíces de las cinco especies evaluadas (Tabla 5).

En Solanum nitidum, los mayores valores de acumulación de plomo, zinc y cadmio fueron obtenidos en la raíces con el tratamiento de $100 \%$ de relave de mina. Acumularon $576 \mathrm{mg}$ de plomo por $\mathrm{kg}^{-1}$ de materia seca (MS), $431.4 \mathrm{mg}$ de zinc $\mathrm{kg}^{-1}$

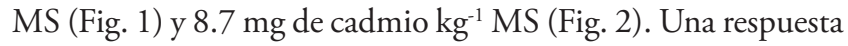
similar fue observada al analizar como plomo total, zinc total y cadmio total, la mayor acumulación de estos tres elementos se dio con el tratamiento de $100 \%$ de relave de mina.

En Brassica rapa, los mayores valores de acumulación de plomo, zinc y cadmio fueron obtenidos en las raíces con el tra- tamiento de $100 \%$ de relave de mina. La especie acumuló 758.8 $\mathrm{mg} \mathrm{kg}^{-1} \mathrm{MS}$ de plomo, $550 \mathrm{mg} \mathrm{kg}^{-1} \mathrm{MS}$ de zinc (Fig. 3) y $4.9 \mathrm{mg}$ $\mathrm{kg}^{-1} \mathrm{MS}$ de cadmio (Fig. 4). Una tendencia similar fue observada cuando se analizó como plomo total, zinc total y cadmio total, donde la mayor acumulación de estos tres elementos se generó con el tratamiento de $100 \%$ de relave de mina.

En Fuertesimalva echinata, los mayores valores de acumulación de plomo, zinc y cadmio fueron obtenidos también en las raíces con el tratamiento de $100 \%$ de relave de mina. Acumularon $2015.1 \mathrm{mg} \mathrm{kg}^{-1} \mathrm{MS}$ de plomo, $1024.2 \mathrm{mg} \mathrm{kg}^{-1}$ MS de zinc (Fig. 5), y $11 \mathrm{mg} \mathrm{kg}^{-1} \mathrm{MS}$ de cadmio (Fig. 6). Una tendencia similar fue observada cuando se analizó como plomo total, zinc total y cadmio total, donde la mayor acumulación de estos tres elementos se generó con el tratamiento de 100\% de relave de mina.

De todas las especies altoandinas evaluadas, esta especie posee la mayor eficacia de fitoextraer y acumular plomo y zinc estas características ameritan considerala como una especie fitorremediadora de suelos contaminadas con estos elementos.

En Urtica urens, los mayores valores de acumulación de plomo, zinc y cadmio fueron obtenidos en las raíces con el tratamiento de $100 \%$ de relave de mina. Acumularon $854.5 \mathrm{mg}$ de plomo $\mathrm{kg}^{-1} \mathrm{MS}, 452.8 \mathrm{mg}$ de zinc $\mathrm{kg}^{-1} \mathrm{MS}$ (Fig. 7) y $8.9 \mathrm{mg}$ de cadmio $\mathrm{kg}^{-1}$ MS (Fig. 8). Una tendencia similar fue observada cuando se analizó como plomo total, zinc total y cadmio total, en donde la mayor acumulación de estos tres elementos se generó con el tratamiento de $100 \%$ de relave de mina.

En Lupinus ballianus los mayores valores de acumulación de plomo y cadmio fueron obtenidos en las raíces con el tratamiento de $100 \%$ de relave de mina. Acumularon $992.8 \mathrm{mg}$ de plomo $\mathrm{kg}^{-1} \mathrm{MS}$ (Fig. 9) y $287.3 \mathrm{mg}$ de cadmio kg-1 MS (Fig. 10). Una tendencia similar fue observada cuando se analizó como plomo total, zinc total y cadmio total, en donde la mayor acumulación 


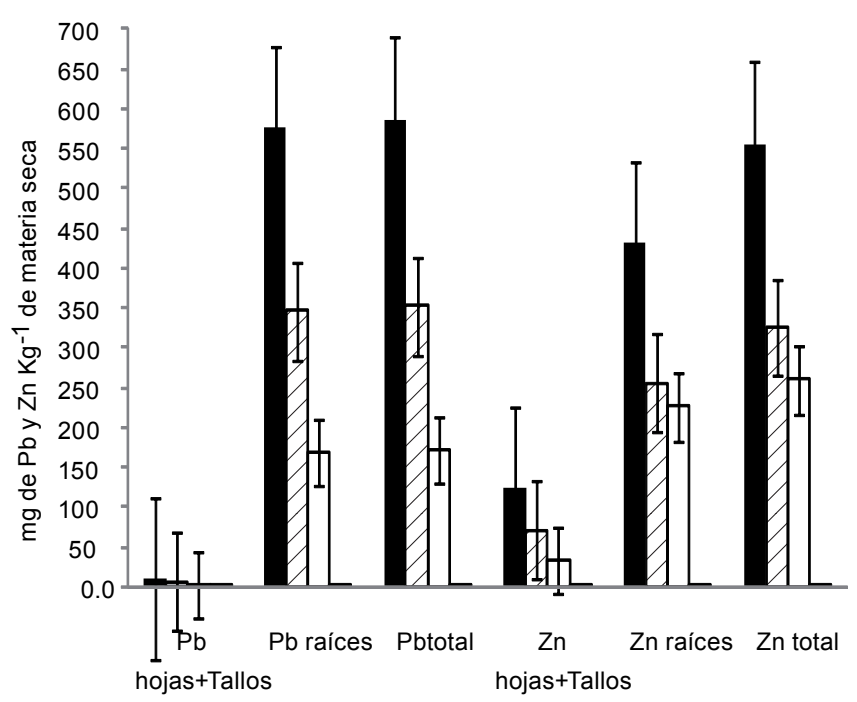

Figura 1. Acumulación de plomo y zinc en hojas y tallos, raíces y plomo y zinc total en Solanum nitidum Ruiz \& Pav.

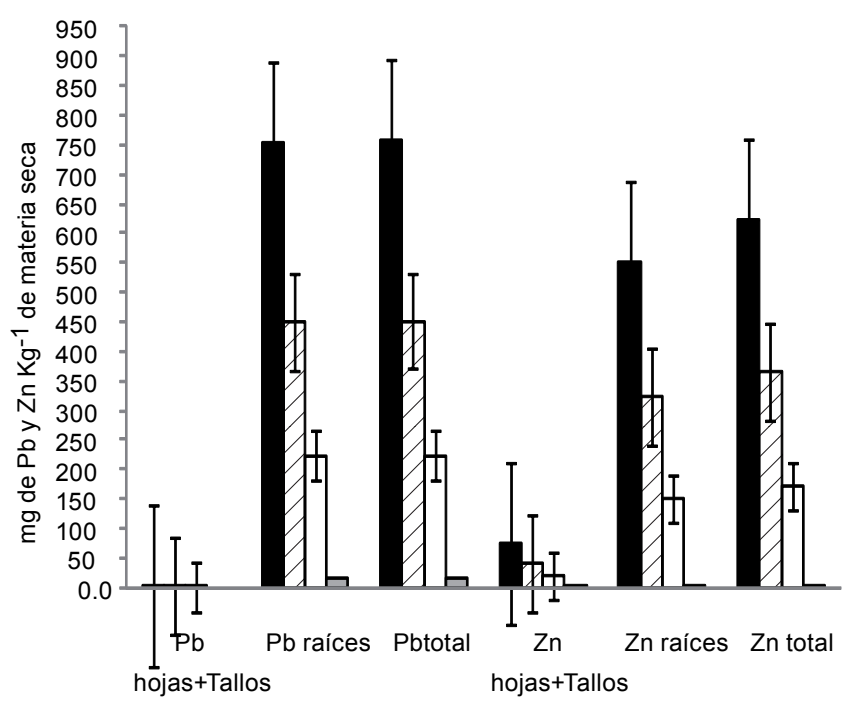

Figura 3. Acumulación de plomo y zinc en hojas y tallos, raíces y plomo y zinc total en Brassica rapa L.

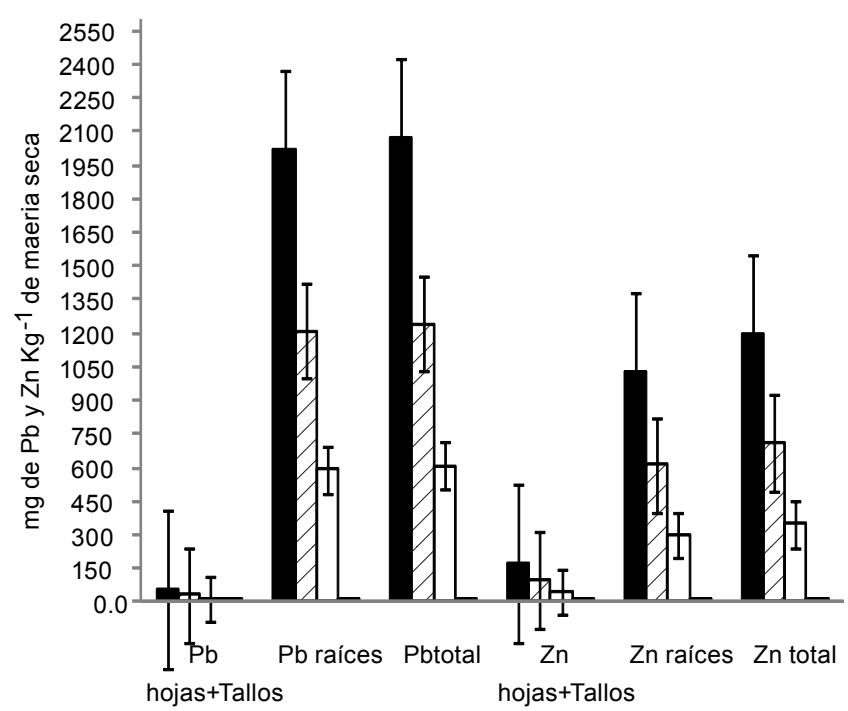

Figura 5. Acumulación de plomo y zinc en hojas y tallos, raíces y plomo y zinc total en Fuertesimalva echinata (C.Presl) Fryxell.

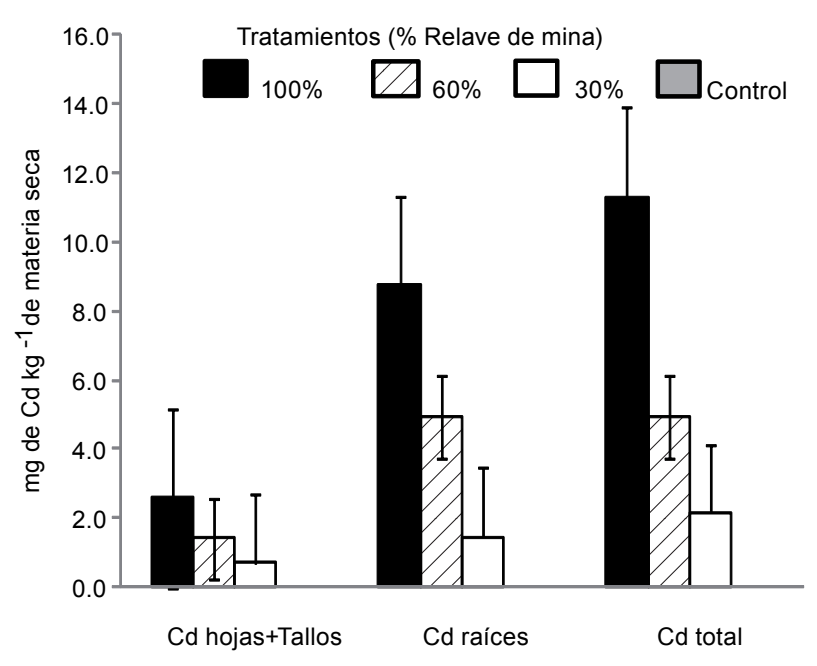

Figura 2. Acumulación de cadmio en hojas y tallos, raíces y cadmio total en Solanum nitidum Ruiz \& Pav.

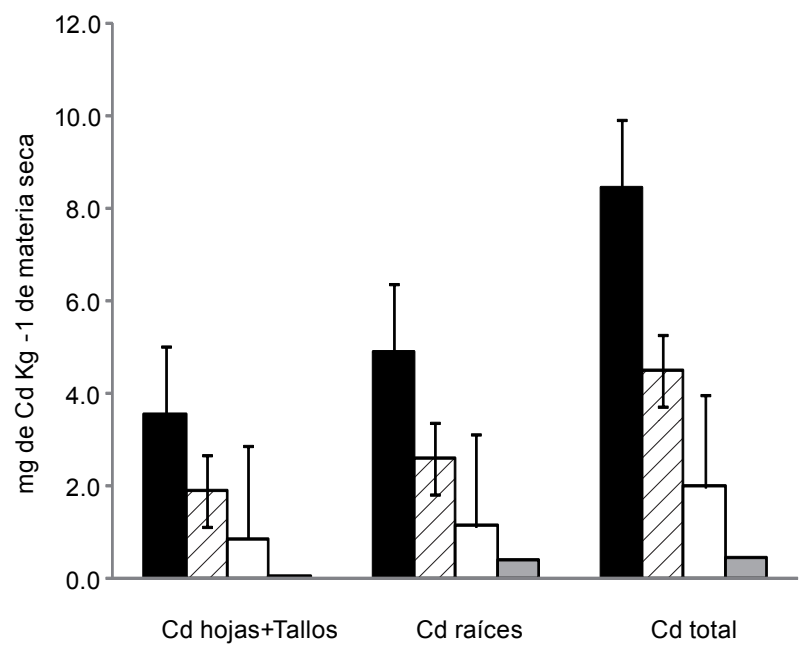

Figura 4. Acumulación de cadmio en hojas y tallos, raíces y cadmio total en Brassica rapa L.

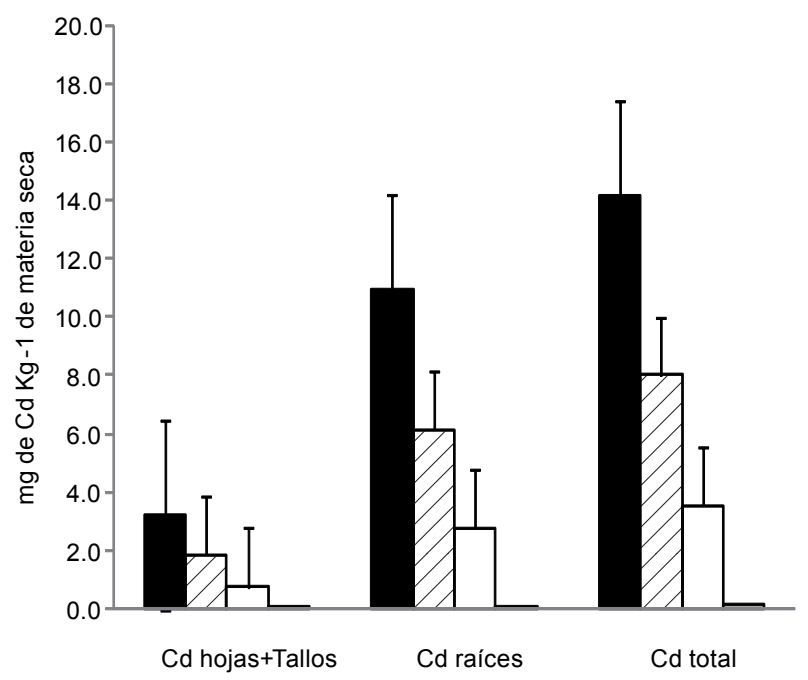

Figura 6. Acumulación de cadmio en hojas y tallos, raíces y cadmio total en Fuertesimalva echinata (C.Presl) Fryxel. 


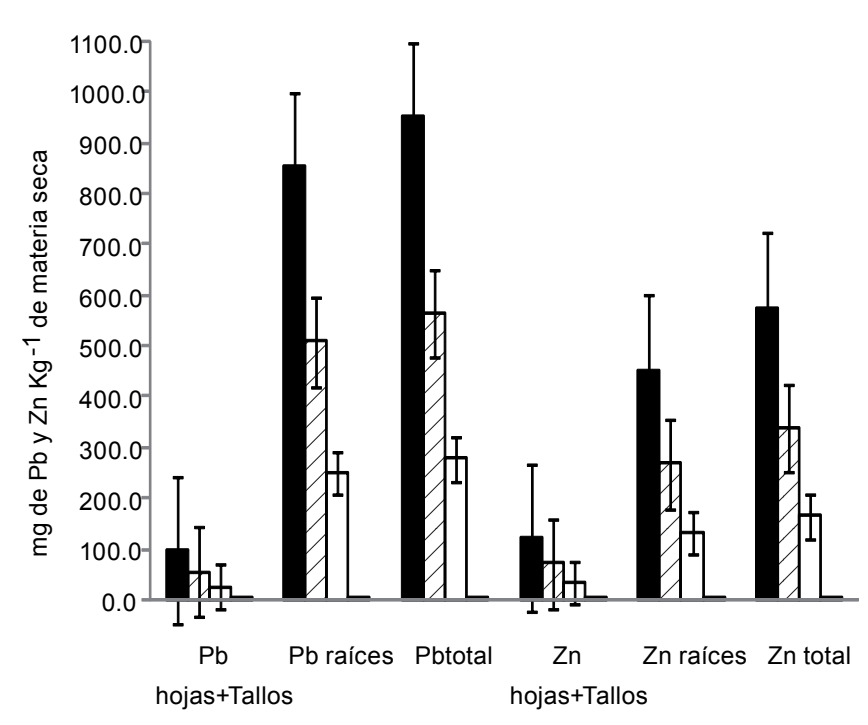

Figura 7. Acumulación de plomo y zinc en hojas y tallos, raíces y plomo y zinc total en Urtica urens $\mathrm{L}$.

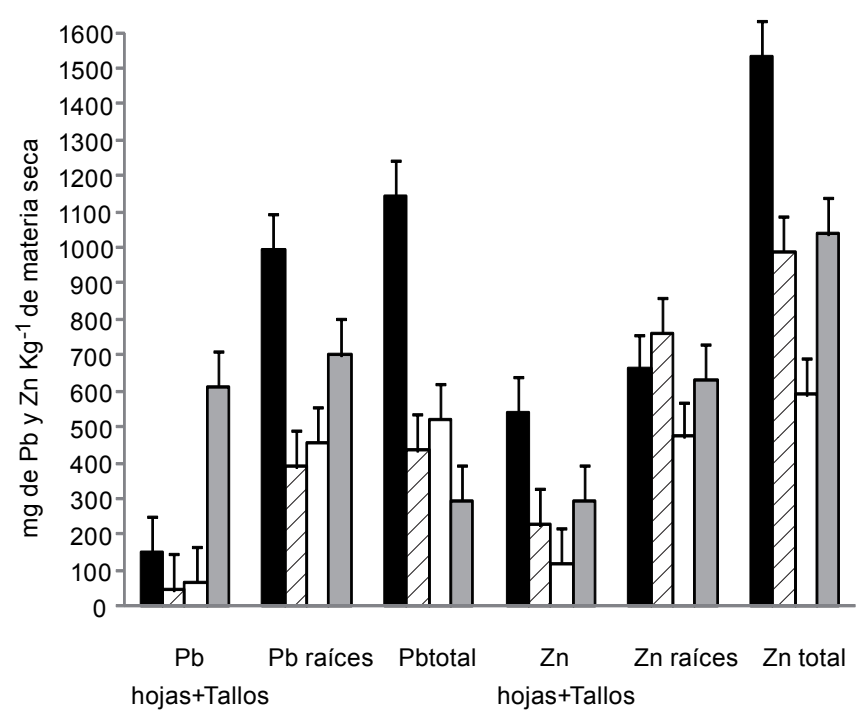

Figura 9. Acumulación de plomo y zinc en hojas y tallos, raíces y plomo y zinc total en Lupinus ballianus C.P. Sm.

de estos tres elementos se generó con el tratamiento de 100\% de relave de mina.

Sin embargo, la mayor acumulación de zinc fue obtenida con el tratamiento de $60 \%$ de relave de mina $\left(763.6 \mathrm{mg} \mathrm{kg}^{-1}\right.$. MS). Una tendencia similar fue observada cuando se analizó como plomo total, zinc total y cadmio total, en donde la mayor

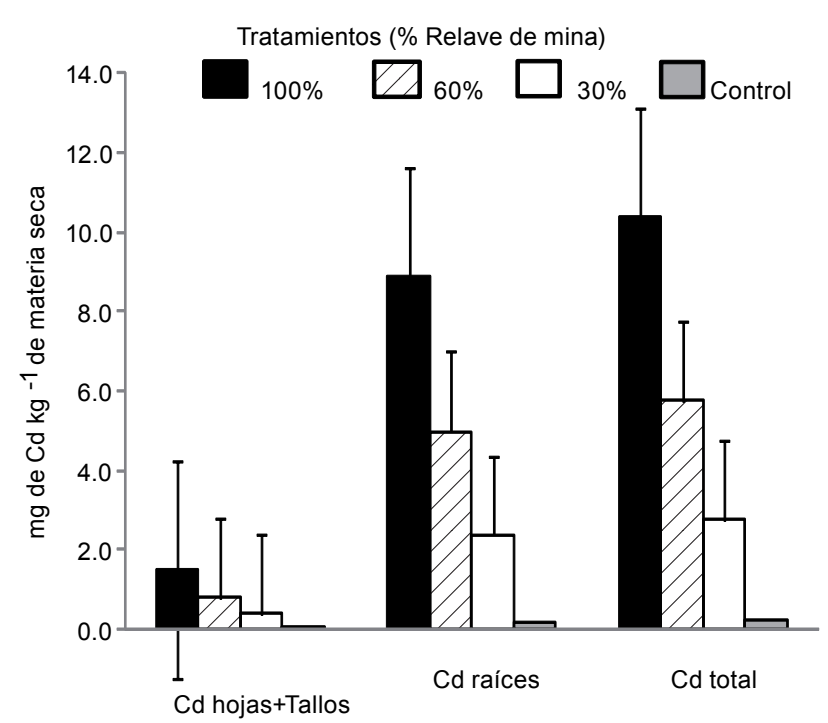

Figura 8. Acumulación de cadmio en hojas y tallos, raíces y plomo y zinc total en Urtica urens $\mathrm{L}$.

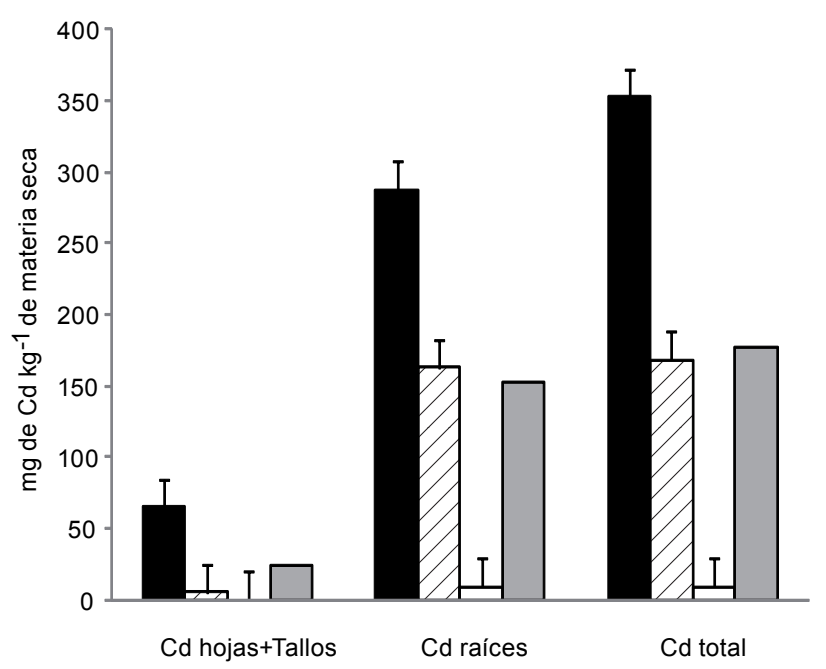

Figura 10. Acumulación de cadmio en hojas y tallos, raíces y plomo y cadmio total en Lupinus ballianus C.P. Sm.

acumulación de estos tres elementos se generó con el tratamiento de $60 \%$ de relave de mina.

Índice de tolerancia de las especies a plomo, zinc y cadmio.- La prueba de comparación múltiple de medias de Tukey, mostró que existen diferencias significativas $(\mathrm{P}<0.05)$ entre las los tratamientos para el IT al plomo, zinc y cadmio calculadas

Tabla 6. Índice de Tolerancia (IT) de cinco especies altoandinas evaluadas con tratamientos de relave de mina (\%) en el Distrito de Lachaqui, Provinvia de Canta, Región Lima.

\begin{tabular}{lcccc}
\hline \multirow{2}{*}{ Especies } & \multicolumn{3}{c}{ Tratamientos (relave de mina) } \\
\cline { 2 - 5 } & $\mathbf{1 0 0 \%}$ & $\mathbf{6 0 \%}$ & $\mathbf{3 0 \%}$ & Control \\
\hline Solanum nitidum Ruiz \&Pav. & $\$ 28.9 \mathrm{~d}$ & $68.5 \mathrm{c}$ & $87.5 \mathrm{~b}$ & $100 \mathrm{a}$ \\
Brassica rapa L. & $37.3 \mathrm{~d}$ & $47.9 \mathrm{c}$ & $63.7 \mathrm{~b}$ & $100 \mathrm{a}$ \\
Fuertesimalva echinata (C.Presl) Fryxel & $41.5 \mathrm{~d}$ & $59.1 \mathrm{c}$ & $80.7 \mathrm{~b}$ & $100 \mathrm{a}$ \\
Urtica urens L. & $32.5 \mathrm{~d}$ & $50.1 \mathrm{c}$ & $58.4 \mathrm{~b}$ & $100 \mathrm{a}$ \\
Lupinus ballianus C.P. Sm. & $20.0 \mathrm{~d}$ & $67.9 \mathrm{c}$ & $77.2 \mathrm{~b}$ & $100 \mathrm{a}$ \\
\hline
\end{tabular}

$\S$ Valores con la misma letra en las columnas son estadísticamente iguales (Tukey, $\alpha=0.05$ ). 
para los cinco especies alto andinas (Tabla 6). Los valores del IT evidencian que Fuertesimalva echinata tiene la mayor capacidad de tolerancia al tratamiento de $100 \%$ de relave de mina, con un IT de $41.5 \%$, pero con una baja producción en biomasa (Tabla 6). Solanum nitidum y Lupinus ballianus presentaron IT mediano al tratamiento de $60 \%$ de relave de mina, logrando obtener $68.5 \%$ y 67.9 de IT, respectivamente.

\section{Discusión}

Biomasa.- De las cinco especies evaluadas, los menores valores de rendimiento de biomasa fueron obtenidos con el tratamiento de $100 \%$ de relave de mina. Por consiguiente, la presencia de altas concentraciones de plomo, zinc, cadmio y otros metales del relave polimetálico se asocian a una baja producción de biomasa, en comparación con el tratamiento control.

Zornoza et al. (2002) indican que la disminución de la biomasa puede atribuirse a la reducción de la longitud de planta y a la pérdida de hojas, pero también podría deberse a la toxicidad originada por la alta concentración de cadmio disponible en el suelo (Ehsan et al. 2007).

Estudios realizados en especies de los géneros Lupinus, Solanum y Brassica siguieron comportamientos similares a los previamente descritos, tal fue el caso de Lupinus uncinatus, el cual cuando fue evaluado con diferentes concentraciones de cadmio aplicado al suelo, presentó inhibición del crecimiento en longitud de planta y en número de hojas; consecuentemente, el cadmio también influyó significativamente en el rendimiento de la materia seca en raíces, hojas y tallos (Ehsan et al. 2009).

En Solanum elaegnifolium, la presencia de plomo en el sustrato, disminuyó la acumulación de materia seca total (Trejo et al. 2009). En otras especies de Brassica evaluadas como Brassica juncea, B. rapa y B. napus, la toxicidad de zinc y cobre, redujo significativamente el crecimiento de sus raíces y el peso seco (Stephen et al. 1997). Finalmente, Diez (2008) indicó que cuando evaluó la resistencia y bioacumulación de zinc en diferentes especies de plantas autóctonas de España, la producción de biomasa en todas las especies estudiadas disminuyó significativamente debido a la toxicidad de este metal.

Acumulación y distribución de Plomo Zincy Cadmio.- En Solanum nitidum, Brassica rapa, Fuertesimalva echinata y Urtica urens los mayores valores de acumulación de plomo y cadmio fueron obtenidos con el tratamiento de $100 \%$ de relave de mina. Con excepción del zinc, donde la mayor concentración de este elemento fue obtenido en Lupinus ballianus, con el tratamiento de $60 \%$ de relave de mina.

Las cinco especies altoandinas evaluadas poseen la estrategia de acumular metales en las raíces, independientemente del nivel de contaminación del sustrato, esta estrategia es realizada por aquellas plantas denominadas fitoestabilizadoras (Hazrat et al. 2013). Estas especies tienen la capacidad de reducir el transporte de los contaminantes al tallo y las hojas, y minimizando la movilidad de los metales pesados mediante la precipitación y la acumulación en las raíces (Alkorta et al. 2004). Se ha comprobado, que las raíces producen cambios en la especiación de metales, al producir variaciones en el potencial redox, secreción de protones, y de agentes quelantes, además gran parte de los iones metálicos son adsorbidos físicamente a las superficies externas de las paredes celulares cargadas negativamente (Diez 2008).
En Solanum nitidum, con el tratamiento $100 \%$ de relave de mina fueron obtenidos los mayores valores de acumulación de plomo (576 mg kg-1 MS), zinc (431.4 $\mathrm{mg} \mathrm{kg}^{-1} \mathrm{MS}$ ) y cadmio (8.7 $\mathrm{mg} \mathrm{kg}^{-1} \mathrm{MS}$ ). Las concentraciones de zinc y cadmio obtenidos, estuvieron muy cercanas a las concentraciones obtenidas por Lerma (2006), en especies vegetales con potencial de acumulación de metales pesados, y donde las raíces de Solanum eleagnifolium acumularon $718.7 \mathrm{mg} \mathrm{kg}^{-1} \mathrm{MS}$ de zinc y 14.5 $\mathrm{mg} \mathrm{kg}^{-1}$ de cadmio. Sin embargo, Trejo et al. (2009) al evaluar la fitoextracción de esta misma especie, obtuvieron concentraciones de plomo en los tejidos entre 3.8 y $6.9 \mathrm{mg} \mathrm{kg}^{-1} \mathrm{MS}$, y la concentración de cadmio varió de 0.2 a $0.3 \mathrm{mg} \mathrm{kg}^{-1} \mathrm{MS}$; de tal manera que la concentración de plomo obtenida en Solanum nitidum, fue superior a la obtenida por Solanum eleagnifolium.

También es importante mencionar que Peng et al. (2006) encontraron que Solanum nigrum acumulaba 99 mg de cadmio $\mathrm{kg}^{-1} \mathrm{MS}$, por lo que es posible que Solanum nitidum sea una planta fito estabilzador a de plomo, zinc y cadmio.

En Brassica rapa, los análisis químicos indicaron que los mayores valores de acumulación de plomo, zinc y cadmio fueron obtenidas en las raíces con el tratamiento de 100\% de relave de mina. Sin embargo, se conoce que especies de otros géneros de brassicáceas fitoextraen y almacenan muchos metales pesados en las hojas, por ejemplo, Thlaspi caerulescens es una especie hiperacumuladora de zinc, que logró acumular hasta $14000 \mathrm{mg} \mathrm{kg}^{-1} \mathrm{MS}$ (Becerril et al. 2007). Asimismo, cuando se cultivaron accesiones seleccionadas de Brassica juncea, B. napus, y de $B$. rapa en suelos contaminados con zinc y cadmio, éstas fueron las más eficientes en la eliminación de zinc fundamentalmente, logrando producir 10 veces más biomasa de tallos y hojas que, T. caerulescens (Ebbs et al. 1997). Además, en el trabajo realizado por Turan y Esringü (2007) en la evaluación de la fitorremediación de Brassica napus y Brassica juncea en suelos contaminados con cobre, cadmio, plomo y zinc, con la adición del quelato EDTA se obtuvieron diferencias significativas entre especies y por órganos, Brassica napus fue la más eficiente en la absorción de cobre, cadmio, plomo y zinc; y en ambas especies la mayor acumulación de metales pesados se obtuvo en las raíces.

En Fuertesimalva echinata, los mayores valores de acumulación de plomo (2015.1 $\mathrm{mg} \mathrm{kg}^{-1} \mathrm{MS}$ ), zinc (1024.2 $\mathrm{mg} \mathrm{kg}^{-1} \mathrm{MS}$ ) y de cadmio (11 mg kg-1 MS) fueron obtenidos en las raíces con el tratamiento $100 \%$ de relave de mina. Esta especie altoandina presentó la mayor capacidad de fitoestabilización de plomo y cadmio entre las cinco evaluadas.

Trabajos realizados con otras especies de malváceas corroboran estos resultados, puesto que por ejemplo en Hibiscus cannabinus, la aplicación de abono orgánico promovió una mayor capacidad de acumulación de plomo, y mayor producción de biomasa. Las raíces acumularon más de $85 \%$ del plomo total, indicando que la raíz podría ser una fuente importante de plomo biodisponible (Ho et al. 2008). Brachiaria reptans y Malvastrum coromandelianum fueron las más adecuadas en la fitoestabilización de suelos contaminados con plomo y cobre (Nassir et al. 2011).

Abe et al. (2008) al evaluar la acumulación de cadmio en tallos y raíces de 93 especies vegetales encontraron que Sida rhormbifolia presentó concentraciones de cadmio de $111.9 \mathrm{mg}$ $\mathrm{kg}^{-1} \mathrm{MS}$ en raíces y $23.8 \mathrm{mg} \mathrm{kg}^{-1} \mathrm{MS}$ en tallos, Sida spinosa (46 
mg kg-1 MS en raíces y $8.7 \mathrm{mg} \mathrm{kg}^{-1} \mathrm{MS}$ en tallos), Malva sylvestris (37.9 $\mathrm{mg} \mathrm{kg}^{-1} \mathrm{MS}$ en raíces y $33.5 \mathrm{mg} \mathrm{kg}^{-1} \mathrm{MS}$ en tallos) y Abutilon theophrasti $\left(35.8 \mathrm{mg} \mathrm{kg}^{-1} \mathrm{MS}\right.$ en raíces y $14.9 \mathrm{mg} \mathrm{kg}^{-1}$ MS en tallos). Las concentraciones indicadas de cadmio fueron mayores a las obtenidas en F echinata. No obstante, también De Haro et al. (2000), al evaluar 96 especies de las zonas agrícolas adyacentes a la mina de Aznalcóllar (Espańa), indicaron que Lavatera cretica por ejemplo obtuvo la mayor acumulación de cadmio, y la consideraron como una especie promisoria para fines de fitorremediación.

En Urtica urens, los mayores valores de acumulación de plomo, zinc y cadmio fueron obtenidos en las raíces con el tratamiento de $100 \%$ de relave de mina, acumulando 854.5 mg de plomo kg-1 MS, $452.8 \mathrm{mg}$ de zinc kg-1 MS y $8.9 \mathrm{mg}$ $\mathrm{kg}^{-1}$ MS de cadmio. Por otro lado, el trabajo de Malizia et al. (2012) en Urtica dioica señaló que esta especie acumuló plomo principalmente en hojas, y podría utilizarse en la fitorremediación de suelos contaminados con plomo (Grubor 2008). Sin embargo, en el trabajo de Ziedler (2005), se dio a conocer que Urtica dioica acumulaba cadmio $2,5 \mathrm{mg} \mathrm{kg}^{-1} \mathrm{MS}$ en raíces, 2,8 $\mathrm{mg} \mathrm{kg}^{-1} \mathrm{MS}$ en tallos y 2,1 $\mathrm{mg} \mathrm{kg}^{-1} \mathrm{MS}$ en hojas; de plomo 0,7 $\mathrm{mg} \mathrm{kg}{ }^{-1}$ MS en hojas, $0,6 \mathrm{mg} \mathrm{kg}^{-1}$ MS en raíces y $1,9 \mathrm{mg} \mathrm{kg}^{-1}$ MS en tallos; y de zinc, $0,4 \mathrm{mg} \mathrm{kg}^{-1} \mathrm{MS}$ en hojas, $0,7 \mathrm{mg} \mathrm{kg}^{-1}$ $\mathrm{MS}$ en raíces y $0,9 \mathrm{mg} \mathrm{kg}^{-1} \mathrm{MS}$ en tallos. En el presente trabajo la acumulación de plomo y cadmio obtenidas por Urtica urens, superan muy significativamente a los resultados obtenidos por Ziedler (2005) en $U$. dioica.

En Lupinus ballianus, los mayores valores de acumulación de plomo y cadmio fueron obtenidos en las raíces con el tratamiento de $100 \%$ de relave de mina, acumulando $992.8 \mathrm{mg}$ de plomo kg-1 MS y $287.3 \mathrm{mg}$ de cadmio $\mathrm{kg}^{-1} \mathrm{MS}$. De las cinco especies evaluadas, L. ballianus obtuvo la mayor eficiencia de fitoextracción y acumulación de cadmio.

Ximénez-Embún et al. (2001) al evaluar la acumulación de metales pesado en L. albus, L. luteus, L. angustifolius y en L. hispanicus, mencionó que también se obtuvieron concentraciones más altas de $\mathrm{Pb}$ (II), Cr (III), y Cd (II) en las raíces que en los tallos. Aunque la mayor acumulación de zinc $\left(763.6 \mathrm{mg} \mathrm{kg}^{-1} \mathrm{MS}\right)$ en L. ballianus fue obtenido con el tratamiento de $60 \%$ de relave de mina, Pastor et al. (2003) informan que en la evaluación de la acumulación de zinc en Lupinus albus en suelos contaminados con este metal, la aplicación de 300 partes por millón de zinc produjo desbalances nutricionales, por lo que se obtuvo una alta acumulación de zinc en raíces ( $4640 \mathrm{mg} \mathrm{kg}^{-1} \mathrm{MS}$ ) y en la parte aérea $\left(3605 \mathrm{mg} \mathrm{kg}^{-1} \mathrm{MS}\right)$ y recomiendan su uso potencial en la fitorremediación de suelos ácidos o neutros contaminadas con zinc y en la revegetación de áreas degradadas. La acumulación de zinc en $L$. ballianus fue menor en comparación a los resultados obtenidas por Pastor et al. (2003). Sin embargo, Martínez-Alcalá et al. (2009), indicaron que en los tallos de L. albus observaron una limitada transferencia de los metales pesados, confirmando el uso potencial de esta especie en la fitoinmovilización de los metales pesados, y particularmente en suelos contaminados alcalinos y neutros.

Vásquez et al.(2006) recomiendan el uso de L. albus en la fitoestabilización de suelos contaminados con cadmio y arsénico, y en la revegetación de suelos contaminados por metales. Dary et al. (2010) al evaluar la fitoestabilización in situ de suelos contaminados con metales pesados con el uso de L. luteus, señalan que ésta especie logró acumular rápidamente cobre y cadmio principalmente en la raíces, e indican su uso potencial en la fitoestabilización de metales en el suelo. En el trabajo realizado con L. ballianus se obtuvo una alta eficiencia de acumulación de cadmio en las raíces, lo que ha coincidido con evaluaciones previas realizadas a otras especies del género Lupinus.

Índice de tolerancia de las especies a plomo, zinc y cadmio.- Fuertesimalva echinata fue la especie que obtuvo el mayor índice de tolerancia (IT) al tratamiento de 100\% de relave de mina, con un IT de $41.5 \%$, pero obtuvo una baja acumulación de biomasa. Sin embargo, Solanum nitidum y Lupinus ballianus presentaron IT mediano con índices de 68.5 y $67.9 \%$, respectivamente, con el tratamiento de $60 \%$ de relave de mina. Ehsan et al. (2009) al evaluar la fitoestabilización con Lupinus uncinatus de suelos contaminados con cadmio señalan que cuando son aplicadas concentraciones de 9 y $18 \mathrm{mg} \mathrm{kg}^{-1} \mathrm{de}$ este metal al suelo no se afectó considerablemente la tolerancia a los metales y se obtuvieron índices de tolerancia de 88 y $82 \%$, respectivamente; mientras que con la concentración de $27 \mathrm{mg}$ $\mathrm{kg}^{-1}$ de cadmio observaron una disminución considerable de la tolerancia, afectándose significativamente la altura de planta (disminución) y el número de hojas.

Los valores calculados para L. ballianus se aproximaron a los resultados de Ehsan et al. (2009). Aunque, Ximénez-Embún et al. (2002) señaló que Lupinus albus cultivado durante 4 semanas en arena contaminada con $50 \mathrm{mg}$ de cadmio $\mathrm{L}^{-1}$ obtuvo un índice de tolerancia a metales cerca al $100 \%$. Por otro lado Diez (2008) obtuvo un índice de tolerancia de un $42 \%$ para Cytisus scoparius con sustratos fuertemente contaminado con zinc y en tratamientos moderadamente contaminados de zinc, obtuvo un IT de 85\%. Gisbert et al. (2006) al evaluar la acumulación y la tolerancia a metales pesados de especies de brassicáceas cultivadas en suelos contaminados del mediterráneo, obtuvieron IT de 97.6\% para Brassica juncea, IT de $74.2 \%$ para B. carinata cultivar 117 , IT de $66.5 \%$ en B. carinata cultivar 2920 ; IT de $66.6 \%$ B. oleracea y IT de $72 \%$ para Hirschfeldia incana, respectivamente.

En conclusión, la producción de biomasa en las cinco especies disminuyó significativamente con el tratamiento de $100 \%$ de relave de mina (RM). En Solanum nitidum, Brassica rapa, Fuertesimalva echinata, Urtica urens y Lupinus ballianus, los mayores valores de acumulación de plomo y cadmio fueron obtenidas en las raíces, con el tratamiento de 100\% RM debido al proceso de fitoestabilización. En Fuertesimalva echinata la más alta acumulación de plomo y de zinc fue obtenido en las raíces, con $2015.1 \mathrm{mg} \mathrm{kg}^{-1}$ de materia seca (MS) y $1024.2 \mathrm{mg}$ $\mathrm{kg}^{-1}$ MS, respectivamente con el tratamiento de 100\% RM. En Lupinus ballianus la más alta acumulación de cadmio fue obtenido en raíces con $287.3 \mathrm{mg} \mathrm{kg}^{-1} \mathrm{MS}$ con el tratamiento de $100 \%$ RM. Pero la mayor acumulación de zinc fue obtenido con el tratamiento de 60\% RM. Fuertesimalva echinata obtuvo el mayor índice de tolerancia (IT) al tratamiento de $100 \%$ RM, con un IT de 41.5\%, Solanum nitidum y Lupinus ballianus presentaron un IT mediano con índices de $68.5 \mathrm{y}$ $67.9 \%$, respectivamente.

\section{Agradecimientos}

Agradecemos a la Dra. Mónica Arakaki Makishi por la revisión del manuscrito. 


\section{Literatura citada}

Abe T., M. Fukami \& M. Ogasawara. 2008. Cadmium accumulation in the shoots and roots of 93 weed species. Soil Science and Plant Nutrition, 54, 566-573. doi: 10.1111/j.17470765.2008.00288.x.

Adriano D.C. 2001. Trace elements in terrestrial environments: Biogeochemistry, Bioavailability and Risks of Metals. 2nd Edition. Springer-Verlag New York. Berlin Heidelberg. http://dx.doi.org/10.1007/978-0-387-21510-5

Alkorta I., I. Becerril \& C. Garbisu. 2010. Phytostabilization of metal contaminated soils. Reviews on Environmental Health, 25: 135-146. http://dx.doi.org/10.1515/REVEH.2010.25.2.135

Alkorta I., J. Hernández-Allica, J.M. Becerril, I. Amezaga, I. Albizu \& C. Garbisu. 2004. Recent findings on the phytoremediation of soils contaminated with environmentally toxic heavy metals and metalloids such as zinc and cadmium and arsenic. Rev. Environ. Sci. Bio/Technology 3: 71-90. doi:10.1023/B:RESB.0000040059.70899.3d.

Allan J.E. 1971. The preparation of agricultural samples for analysis by atomic absorption spectroscopy. Varian Techtron, Walnut Greek, California. USA.

Baker A.J.M. \& J Proctor. 1990. The influence of cadmium, copper, lead and zinc on the distribution and evolution of metallophytes in the British Isles. Plant Systematics and Evolution, 173: 91-108. Doi 10.1007/BF00937765

Baker A.J.M., S.P Mc Grath., R.D. Reeves \& J.A.C. Smith. 2000. Metal hyperaccumulator plants: a review of the ecology and physiology of a biological resource for phytoremediation of metal-polluted soils. In: Terry, N. \& Bañuelos, G.S. (eds.), Phytoremediation of Contaminated Soil and Water, pp. 85-108. Lewis Publishers, Boca Raton

Barceló J \& C. Poschenrieder. 2003. Phytoremediation: principles and perspectives. Contrib. Sci. 2, 333-344.

Becerril J.M., O. Barrutia, J.I. García Plazaola, A. Hernández, J.M. Olano \& C. Garbisu. 2007. Especies nativas de suelos contaminados por metales: aspectos ecofisiológicos y su uso en fitorremediación. Ecosistemas 16 (2): 50-55.

Brooks R.R., J. Lee, R.D. Reeves \& T. Jaffré. 1977. Detection of metalliferous rocks by analysis of herbarium specimens of indicator plants. Journal of Geochemical Exploration, 7: 49-77. http://dx.doi.org/10.1016/0375-6742(77)90074-7

Chaney R.L. 1983. Plant uptake of inorganic waste constituents. In: J.F. Parr, P.B Marsh, J.M. Kla (eds.) Land treatment of hazardous wastes, pp. 50-76. Park Ridge, NJ, USA: Noyes Data Corporation.

De Haro A., A. Pujadas, A. Polonio, R. Font, D. Vélez, R. Montoro $\&$ M. del Rio, M. .2000. Phytoremediation of the polluted soils after the toxic spill of the Aznalcóllar mine by using wild species collected in situ. Fresenius Environmental Bulletin. 9(5/6):275-280.

De La Cruz-Landero N., V.E. Hernández, E. Guevara, M.A. López, A.T. Santos, E. Ojeda-Trejo \& A. Alderete-Chávez. 2010. Lupinus versicolor response in soils contaminated with heavy metals from a petroleum extraction field. J. Applied Sci., 10: 694-698. Doi: 10.3923/jas.2010.694.698.

Diez F.J. 2008. Fitocorrección de suelos contaminados con metales pesados. Evaluación de plantas tolerantes y optimización del proceso mediante prácticas agronómicas. Tesis Doctoral. Universidad Santiago de Compostela, España.331 p.

Ebbs S.D., M. Lasat, D.J. Brady, J. Cornish, R. Gordonand \& L.V. Kochian. 1997.Phytoextraction of Cadmium and Zinc from a Contaminated Soil. Journal of Environmental Quality. 26(5): 1424-1430. http://dx.doi.org/10.2134/ jeq1997.2651424x

Ehsan M., K. Santamaría-Delgado, A. Vázquez-Alarcón, et al. 2009. Phytostabilization of cadmium contaminated soils by Lupinus uncinatus Schdl. Journal of Agricultural Research 7(2): 390-397. www.inia.es/sjar.

Ehsan M., P.A. Molumeli, H.V Espinosa, R.A. Baeza, M.J. Perez, H.M. Soto, T.E. Ojeda, D. Jaen, B.A. Ruiz \& S.E. Robledo, 2007. Contamination time effect on plant available fractions of cadmium and zinc in a mexican clay loam soil. J. Appl Sci 7(16): 2380-2384. http://dx.doi.org/10.3923/ jas.2007.2380.2384
Ghosh M. \&, S.P Singh. 2005. A review on phytoremediation of heavy metals and utilization of it's by products. Appl. Ecol. Environ. Res. 3(1): 1-18. http://dx.doi.org/10.15666/ aeer/0301_001018

Gisbert CR., J. Clemente, J. Navarro-Aviñó, C. Baixauli, et al. 2006. Tolerance and accumulation of heavy metals by Brassicaceae species grown in contaminated soils from Mediterranean regions of Spain. Environmental and Experimental Botany.56, (1):19-27. doi:10.2134/ jeq1986.00472425001500030002x.

Grubor M. 2008. Lead uptake, tolerance, and accumulation exhibited by the plants Urtica dioica and sedum spectabile in contaminated soil without additives. Arch. Biol. Sci. 60 (2), 239-244. Doi :10.2298/AbS0802239G

Hazrat A, E. Khan \& M.A Sajad. 2013. Phytoremediation of heavy metals concepts and applications. Chemosphere (91): 869-881. Doi 10.1007/s11270-013-1863-z.

Ho WM., L.H. Ang \& D. Lee. 2008. Assessment of Pb uptake, translocation and immobilization in kenaf (Hibiscus cannabinus L.) for phytoremediation of sand tailings. Journal of Environmental Sciences, 20 (11):1341-1347. http://dx.doi. org/10.1016/S1001-0742(08)62231-7

Lerma I E.M. 2006. Evaluación de Suelos y especies vegetales con potencial de acumulación de metales pesados. Tesis de Maestría. Universidad Autónoma de Chihuahua. México.

Malizia D., A. Giuliano, G. Ortaggi \& A. Masotti. 2012. Common plants as alternative analytical tools to monitor heavy metals in soil. Chemistry Central Journal. 6 (2): 1-10

Nazir A. R. Nassem, M. Ajaib, N. Khan \& M.F. Siddiqui. 2011. Hyperaccumulators of heavy metals of industrial areas of slamabad and Rawalpindi. Pak. J. Bot., 43(4): 1925-1933.

Pastor J., A.J. Hernández, N. Prieto \& M. Fernández-Pascual. 2003. Accumulating behavior of Lupinus albus L. growing in a nor$\mathrm{mal}$ and a decalcified calcic luvisol polluted with $\mathrm{Zn}$. Plant Physiol 160, 1457-1465. http://dx.doi.org/10.1078/0176$1617-01007$

Peng K, X.D. Li, C.L. Luo \& Z. Shen. 2006. Vegetation Composition and Heavy Metal Uptake by Wild Plants at Three Contaminated Sites in Xiangxi Area, China. Journal of Environmental Science and Health, 41(1):65-76. http:// dx.doi.org/10.1080/10934520500298838

Rascio N \& F. Navari-Izzo. 2011. Heavy metal hyperaccumulating plants: how and why do they do it? And what makes them so interesting? Plant Sci. 180(2):169-81. Doi: 10.1016/j. plantsci.2010.08.016. Epub 2010 Sep 15.

Salt D.E., R.D. Smith \& Y. Raskin.1998. Phytoremediation, En: Annu. Rev. plant. Physiol. Plant. Mol Biol., 49: 643- 668. http:// dx.doi.org/10.1146/annurev.arplant.49.1.643

Trejo C.R., O. Esquivel, A. Pedroza, J.G. Arreola, et al. 2009. Evaluación de Trompillo (Solanum elaeagnifolium) en la fitoextracción de plomo y cadmio en suelos contaminados. Revista Chapingo, 8:247-253.

Turan M \& A. Esringü. 2007. Phytoremediation based on canola (Brassica napus L.) and Indian mustard (Brassica juncea L.) planted on spiked soil by aliquot amount of $\mathrm{Cd}, \mathrm{Cu}, \mathrm{Pb}$, and Zn. Plant Soil Environ., 53 (1): 7-15.

Watson C., I.D. Pulford \& D. Riddell-Black. 2003. Screening of willow species for resistance to heavy metals: comparison of performance in a hydroponics system and field trials. International Journal of Phytoremediation, 5: 351-365. Doi: $10.1080 / 15226510309359042$.

Wenzel W.W., M Bunkowski, M. Puschenreiter, O. Horak. 2003. Rhizosphere characteristics of indigenously growing nickel hyperaccumulator and excluder plants on serpentine soil. Environmental Pollution, 123(1): 131-138. http://dx.doi. org/10.1016/S0269-7491(02)00341-X.

Wong M. H., 2003. Ecological restoration of mine degraded soils, with emphasis on metal contaminated soils. Chemosphere 50: 775-780. http://dx.doi.org/10.1016/S00456535(02)00232-1.

Ximénez-Embún P, Y. Madrid-Albarrán, C. Cámara, et al. 2001. Evaluation of Lupinus Species to Accumulate Heavy Metals from Waste Waters. International Journal of Phytoremediation, 3(4): 369-379. http://dx.doi. org/10.1080/15226510108500065 
Ximénez-Embún P., B. Rodríguez-Sanz, Y. Martínez-Albarrán \& C. Cámara. 2002. Uptake of heavy metals by lupin plants in artificially contaminated sand: preliminary results. Int. Environ Anal Chems, 82: 805-813. Doi: 10.1080/0306731021000102275.

Ziedler, M. 2005. Heavy in two herb species (River Morava, Czech Republic). Polish Journal of Ecology 53 (2): 185-195.
Young K.R., B. León \& A. Cano. 1997. Peruvian Puna. In S. D. Davis,V. H. Heyewood, O. Herrera-Macbride, J. Villa-Lobos and A.C. Hamilton (Eds.), Center of Plant Diversity. A Guide and Strategy for their Conservation. Volume 3, The Americas. The World Wide Fund and IUCN-The World Conservation Union. 470-476.

Zornoza P, S. Vásquez, E. Esteban, M. Fernández, R.Carpena. 2002. Cadmium stress in nodulated white lupin. Strategies to avoid toxicity. Plant Physiol Biochem 40: 1003-1009. http:// dx.doi.org/10.1016/S0981-9428(02)01464-X. 